\title{
Síntesis de Fluoritas de Cerio Modificadas con Samario en el Sistema $\mathrm{Ce}_{1-X} \mathrm{Sm}_{X} \mathrm{O}_{2-\delta}$ para Potencial Uso en Pilas de Combustible de Óxido Sólido
}

\author{
Synthesis of Cerium Fluorites Modified with Samarium in the $\mathrm{Ce}_{1-X} \mathrm{Sm}_{X} \mathrm{O}_{2-\delta}$ \\ System for Potential use in Solid Oxide Fuel Cells
}

\author{
J. A. Gómez-Cuaspud ${ }^{a, *}$ \\ J. B. Carda-Castellób
}

Recepción: 08-jun-13

Aceptación: 28-ago-13

\begin{abstract}
Resumen
Mediante una ruta de síntesis de química húmeda, se obtuvieron cuatro materiales cerámicos tipo fluorita basados en el sistema $\mathrm{Ce}_{1-X} \mathrm{Sm}_{X} \mathrm{O}_{2-\delta}(x=0,5,10$ y $15 \mathrm{~mol} \%)$, con el propósito de generar en ellos propiedades fisicoquímicas de importancia para su eventual aplicación como componentes electródicos en pilas de combustible de óxido sólido (SOFC). La caracterización se realizó en diferentes etapas del proceso de síntesis; inicialmente, los precursores metalorgánicos fueron caracterizados por espectroscopia infrarroja (FTIR) y análisis térmico (TGA-DTA), permitiendo determinar la conformación de las especies tipo citrato. Tras la obtención de las fases cristalinas buscadas, los posteriores análisis, por difracción de rayos X (XRD) y microscopia electrónica (SEM-TEM), permitieron determinar la morfología y las características superficiales y texturales consistentes con una serie de agregados nanométricos $(<41 \mathrm{~nm})$ de forma regular, orientados en el plano cristalográfico (111) con distancias interplanares de 0,31 nm. La composición, determinada por microanálisis de rayos X por energía dispersiva (EDS) y fluorescencia de rayos $\mathrm{X}(\mathrm{XRF})$, indicó una excelente concordancia entre las composiciones propuestas y obtenidas. Los análisis de reducción a temperatura programada $\left(\mathrm{TPR}-\mathrm{H}_{2}\right)$ y de espectroscopia de impedancias AC confirmaron la presencia de propiedades relevantes para el empleo de estas cerámicas en pilas tipo SOFC.
\end{abstract}

Palabras clave: Conductividad eléctrica, Fluoritas, Perovskita, Pila de combustible.

\footnotetext{
${ }^{a}$ Grupo de Desarrollo y Aplicaciones de Nuevos Materiales (DANUM), Universidad Pedagógica y Tecnológica de Colombia, Tunja, Boyacá.

${ }^{\text {b}}$ Grupo de Química del Estado Sólido, Departamento de Química Inorgánica y Orgánica, Universitat Jaume I de Castelló, Castellón de la Plana, España.

*Autor de correspondencia: jairo.gomez01@uptc.edu.co
} 


\begin{abstract}
Four ceramics type fluorite were obtained based on $\mathrm{Ce}_{1-X} \mathrm{Sm}_{X} \mathrm{O}_{2-\delta}(x=0,5,10$ and $15 \mathrm{~mol} \%)$, by progress of a wet chemical synthesis route with purpose of create in the material different physicochemical properties of importance for its potential application as electrode components in solid oxide fuel cells (SOFC). The characterization was performed at different stages of synthesis process, initially metalorganic precursors were characterized by infrared spectroscopy (FTIR) and thermal analysis (TGA-DTA), thereby determining the formation of citrate type species. Subsequent analysis after obtention of crystalline phases sought by $\mathrm{X}$ ray diffraction (XRD) and scanning electron microscopy (SEM, TEM), allowed to evaluate morphology, and some surface and textural characteristics consistent with a set of nanosized aggregates $(<41 \mathrm{~nm})$, regularly oriented in the (111) plane, with interplanar distances of $0.31 \mathrm{~nm}$. The composition was determined by microanalysis of energy dispersive X-ray (EDS) and X-ray fluorescence (XRF) indicating an excellent match between proposed and obtained compositions. Analysis of temperature-programmed reduction $\left(\mathrm{TPR}-\mathrm{H}_{2}\right)$ and $\mathrm{AC}$ impedance spectroscopy, confirmed the presence of relevant properties for use of these ceramics in SOFC systems.
\end{abstract}

Key words: Perovskite, Electrical Conductivity,Fluorites, Fuel Cells

\section{Introducción}

Durante esta segunda década del siglo XXI ha crecido el interés por las pilas de combustible, dada su importancia para la producción de energía en varios sectores críticos y sensibles, en una economía mundial basada en el consumo de combustibles fósiles [1,3]; este tipo de dispositivos, similares a las pilas electroquímicas, están diseñados para alimentación continua de combustible, y producen electricidad a partir de una fuente de hidrógeno y de oxígeno, con rendimientos considerables, debido a que no están limitados por el ciclo de Carnot [4, 6]. A pesar de ello, la dificultad y el costo de su producción radican en la obtención de combustibles de alta densidad energética, bajo costo y disponibilidad, al igual que en el desarrollo de nuevos componentes electródicos que permitan mejorar su vida útil $[7,12]$.

En los últimos años se han desarrollado nuevas técnicas para obtener materiales avanzados de bajo costo y de fácil escalamiento industrial para uso en pilas de combustible; entre ellos, materiales oxídicos basados en estructuras tipo fluorita, en específico, óxidos de cerio, que han recibido especial atención [14] debido a sus notables ventajas, principalmente sustituidos con samario, dada su buena estabilidad térmica y su comportamiento redox, que lo han convertido en un material promisorio para uso como componente anódico en reacciones de oxidación directa de metano. La incorporación de samario en composiciones basadas en óxido de cerio ha proporcionado densidades de potencia de hasta $0,35 \mathrm{Wcm}^{2}$ a temperaturas de $570{ }^{\circ} \mathrm{C}$ y operadas con hidrógeno, en comparación con otros cationes de tierras raras. A pesar de ello, problemas relacionados con el control y la estabilidad de las composiciones, así como con algunas propiedades texturales y parámetros de procesamiento que permitan mantener las propiedades superficiales del material, en orden a mejorar sus características catalíticas, al tiempo que permitan reducir los efectos de sinterización derivados de los métodos convencionales de síntesis, son aspectos que aún deben corregirse en orden a propiciar un nuevo estado del arte para este tipo de componentes, lo cual se ha mejorado en muchos aspectos por el uso de dopantes o modificantes de tierras raras, al igual que por el empleo de nuevos métodos de síntesis de baja temperatura que permiten conservar la mayoría de propiedades de interés en una amplia gama de aplicaciones [14, 17].

En este sentido, la investigación que se presenta en este artículo tuvo como propósito preparar óxidos de cerio tipo fluorita en cuatro composiciones, basadas en el sistema $\mathrm{Ce}_{1-X} \mathrm{Sm}_{X} \mathrm{O}_{2}(\mathrm{x}=0,5,10$ y 15 mol \%) e identificadas como SDC0, SDC5, SDC10 y SDC15, respectivamente, mediante la implementación de una ruta de química suave de polimerizacióncombustión que involucró la formación de compuestos intermedios de coordinación tipo citrato y su posterior evolución en función de la temperatura hasta la conformación de los respectivos polvos cerámicos. Para este fin, los materiales se caracterizaron por espectroscopia infrarroja (FTIR), análisis térmico (TGA-DTA), fluorescencia de rayos X (XRF), 
difracción de rayos X (XRD), microscopía electrónica de barrido y transmisión (SEM-TEM), análisis de reducción a temperatura programada $\left(\mathrm{TPR}-\mathrm{H}_{2}\right)$ y espectroscopia de impedancias AC, con el fin de identificar puntos clave, que conduzcan a proponer variantes en el proceso para determinar las mejores condiciones de síntesis, en orden a posibilitar su empleo como componentes en pilas de combustible de óxido sólido.

\section{Experimental}

Para la síntesis de los óxidos de cerio se utilizó el correspondiente nitrato de cerio, $\mathrm{Ce}\left(\mathrm{NO}_{3}\right)_{3} \cdot 6 \mathrm{H}_{2} \mathrm{O}$ 99,9\% Merck, ajustándolo a una concentración final de 1,0 M, mientras el nitrato de samario fue obtenido por tratamiento del respectivo óxido, $\mathrm{Sm}_{2} \mathrm{O}_{3}$ 99,9\% Metall Rare Earth Limited, con ácido nítrico $68 \%$ Merck en caliente, ajustándolo a una concentración $0,125 \mathrm{M}$. La concentración de las disoluciones del catión $\mathrm{Ce}^{3+}$ se comprobó mediante valoraciones con EDTA en presencia de indicadores, y del catión $\mathrm{Sm}^{3+}$, por valoración gravimétrica. Las disoluciones de los iones metálicos se dosificaron en un reactor de vidrio dotado con agitación magnética (150 rpm), control de temperatura y reflujo. El orden de adición de los iones se estableció de acuerdo con las correspondientes constantes de hidrólisis, asegurando un contenido total de 0,01 moles de iones metálicos en todos los casos.

De esta forma, se sintetizaron un total de cuatro muestras, que permanecieron en calentamiento simultáneo durante 2 horas a $80^{\circ} \mathrm{C}$; al cabo de $10 \mathrm{mi}$ nutos, se les adicionó una disolución de ácido cítrico 2,0 M en una proporción molar 1,5:1 con relación al contenido total de cationes metálicos, con el fin de facilitar la formación de los respectivos compuestos de coordinación, dando continuidad al reflujo durante 30 minutos; así, la concentración de cada catión en el medio de reacción se estableció conforme a la información registrada en la tabla 1.

Tabla 1. Composición y concentración de cada catión en el volumen final de mezcla de las muestras de $\mathrm{Ce}_{1-X} \mathrm{Sm}_{X} \mathrm{O}_{2-\delta}$.

\begin{tabular}{lcccc}
\hline Muestra & Composición & {$\left[\mathrm{Ce}^{3+}\right]$} & {$\left[\mathrm{Sm}^{3+}\right]$} & Vol. (mL) \\
\hline SDC0 & $\mathrm{CeO} 2$ & $0,601 \mathrm{M}$ & --- & 24,95 \\
SDC5 & $\mathrm{Ce}_{0,95} \mathrm{Sm}_{0,05} \mathrm{O}_{2}$ & $0,450 \mathrm{M}$ & $0,023 \mathrm{M}$ & 27,94 \\
SDC10 & $\mathrm{Ce}_{0,90} \mathrm{Sm}_{0,10} \mathrm{O}_{2}$ & $0,367 \mathrm{M}$ & $0,040 \mathrm{M}$ & 32,61 \\
SDC15 & $\mathrm{Ce}_{0,85} \mathrm{Sm}_{0,15} \mathrm{O}_{2}$ & $0,303 \mathrm{M}$ & $0,053 \mathrm{M}$ & 37,28 \\
\hline
\end{tabular}

El pH del medio se ajustó a 4,0 por adición lenta de una disolución concentrada de amoníaco, con el fin de promover la formación preferente de los compuestos de coordinación tipo citrato y evitar potenciales reacciones de hidrólisis o precipitados insolubles, de acuerdo con la información derivada del análisis y modelamiento propuesto, utilizando el programa Hydra-Medusa [18]. Los soles resultantes se calentaron a $120^{\circ} \mathrm{C}$, durante 24 horas y, luego, a 250 ${ }^{\circ} \mathrm{C}$, hasta el inicio de un proceso de autocombustión que generó una serie de conglomerados de polvos cerámicos, los cuales finalmente se trataron a 800 ${ }^{\circ} \mathrm{C}$ durante 30 minutos para eliminar residuos carbonosos remanentes de la etapa de combustión. El análisis de los precursores se hizo por espectroscopía infrarroja en un equipo Perkin-Elmer FTIR-1000, y los análisis de tipo termogravimétrico, en un equipo simultáneo TG-STDA Mettler Toledo modelo TGA/SDTA/1600, para confirmar la presencia de especies tipo citrato; estos análisis permitieron evaluar la temperatura óptima para la consolidación de la fase cristalina buscada. La composición se confirmó por fluorescencia de rayos $\mathrm{X}$ en un espectrómetro secuencial de rayos $\mathrm{X}$ por dispersión de longitudes de onda S4 Pioneer, Bruker.

La formación de las fases y la morfología de los diferentes sólidos se determinaron por difracción de rayos X, en un equipo PANAlytical X'pert PRO MPD, con detector Ultra fast X'Celerator en disposición Bragg-Brentano, usando la radiación $\mathrm{Cu} \mathrm{K} \alpha$ $(\lambda=1,54186 \AA)$ entre 10 y $75^{\circ} 2 \theta$ con pasos de $0,02^{\circ}$. El análisis microscópico (SEM-TEM) se realizó en un equipo Leica-Zeiss LEO 440 con cañón de electrones: 1-30kV, $\mathrm{La}_{6} \mathrm{y} \mathrm{W}$, con imagen digital y analógica EDS y en un equipo JEOL 2100 con cañón termoiónico de $\mathrm{LaB}_{6}$ con un voltaje de aceleración de $200 \mathrm{kV}$ dotado con cámara CCD de adquisición de imágenes de alta resolución. Para este propósito, las muestras se molturaron hasta la obtención de polvos finos que se tamizaron a malla 200 U.S. estándar y se dispersaron en un tubo de ensayo con 5.0 $\mathrm{mL}$ de agua. Cada tubo se colocó en ultrasonido por un periodo de 30 minutos, al cabo de los cuales se tomó una alícuota de $1.0 \mathrm{~mL}$ y se diluyó hasta 5.0 $\mathrm{mL}$ con agua desionizada, dejándola nuevamente en ultrasonido por un periodo de 5 minutos como base para obtener las placas de microscopía. Finalmente, se tomó una gota de esta disolución y se dejó secar a $45^{\circ} \mathrm{C}$ durante una hora. 
Los resultados de difracción se analizaron y refinaron utilizando el programa X'Pert High Score y Cellref, mientras la determinación del tamaño de cristalito se realizó con la ecuación de Debye-Scherrer, utilizando la señal de difracción más característica por el programa Scherrer Calculator. Los análisis de reducción a temperatura programada $\left(\mathrm{TPR}-\mathrm{H}_{2}\right)$ fueron medidos en un equipo Chembet 300 (Quantachrome) dotado con un detector de conductividad térmica (TCD); para este fin, $50 \mathrm{mg}$ de cada muestra fueron colocados dentro de un tubo de cuarzo en "U" y desgasificados a $400{ }^{\circ} \mathrm{C}$ durante 1 hora con un flujo de argón, manteniendo constante esta temperatura durante 30 minutos. Luego, las muestras fueron enfriadas a temperatura ambiente y estabilizadas en un flujo de argón e hidrógeno $(99,998 \%$ de pureza, 90/10 relación volumétrica). La temperatura y las señales del detector de conductividad térmica fueron continuamente registradas durante el calentamiento a $5{ }^{\circ} \mathrm{C} \cdot \mathrm{min}^{-1}$, desde 25 hasta $1000^{\circ} \mathrm{C}$, para obtener los correspondientes perfiles.

Finalmente, la caracterización eléctrica de los sólidos se realizó mediante espectroscopia de impedancias AC en un equipo tipo Agilent 4294A entre 40 $\mathrm{Hz}$ y $13 \mathrm{MHz}$ a temperatura ambiente; los datos de impedancia se corrigieron por la geometría global de las pastillas y de la celda blanco, mientras un medidor de impedancia LCR, QuadTech 1920, se utilizó para mantener la medición de frecuencia fija en el intervalo de frecuencias escogido.

\section{Resultados y discusión}

La obtención de los soles de citratos de cerio y samario a $120{ }^{\circ} \mathrm{C}$ se realizó durante 24 horas, y su posterior caracterización por espectroscopia infrarroja demostró, entre otras cosas, que la formación de los correspondientes compuestos de coordinación a temperaturas relativamente bajas es efectiva, lo cual es identificable por la separación de la banda del grupo carboxílico libre ( 1730) en dos bandas muy características, llamadas voCOasym $\left(\sim 1587 \mathrm{~cm}^{-1}\right) \mathrm{y}$ $v_{\text {OCOsym }}\left(\sim 1380-1400 \mathrm{~cm}^{-1}\right)$, y por el cambio de la banda asignada a $v_{(\mathrm{C}-\mathrm{OH})}$ a frecuencias más bajas $\left(\sim 1100 \rightarrow 1080-1040 \mathrm{~cm}^{-1}\right)[19,21]$. Adicionalmente, no se encontraron diferencias significativas que permitieran identificar cambios en la conformación y estructura de las especies citrato, formadas en cada uno de los cuatro sistemas propuestos, como se indica en la figura $1[22,24]$.

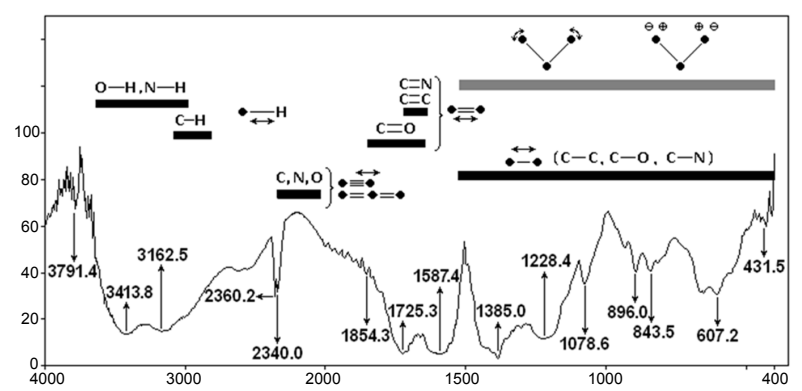

Figura 1. Espectro infrarrojo del precursor de SDC5 que muestra las principales bandas de estiramiento y flexión de los grupos orgánicos presentes en las muestras sintetizadas.

El análisis térmico, entre 25 y $1000{ }^{\circ} \mathrm{C}$, de los precursores obtenidos se realizó con el fin de esclarecer el comportamiento térmico de las muestras sintetizadas y las temperaturas ideales de conformación de la fase buscada. Inicialmente, el estudio demostró un descenso continuo en el peso de la muestra, asociado a una rápida evaporación del agua retenida por adsorción, confirmando que este proceso ocurre en dos etapas plenamente diferenciables; en la primera de ellas, se favorece la desprotonación de los ligandos de grupos $\mathrm{OH}$ entre 50 y $60{ }^{\circ} \mathrm{C}$, mientras que en la segunda se sugiere la eliminación de las moléculas de agua presentes en sus diferentes modos de enlace, y puede terminar entre 150 y $200{ }^{\circ} \mathrm{C}$ [25], hasta la conformación de los respectivos compuestos de coordinación. Posteriormente, se muestra una considerable reacción exotérmica a $165^{\circ} \mathrm{C}$, asociada al proceso de autocombustión, en el cual se da una rápida volatilización de la mayor parte de los componentes orgánicos, registrando pérdidas de peso cercanas al $80 \%$ y dando origen a un conglomerado de precursores amorfos entre 170 y $200^{\circ} \mathrm{C}$. Es claro que, si bien el tipo de coordinación de los grupos activos del ácido cítrico se modifica con el incremento de la temperatura, y la naturaleza de la disolución de partida está en continuo cambio, esta puede generar un incremento moderado de la entalpía asociada a los procesos de evaporación de agua. En una primera etapa de reacción prevalecen ligandos unidentados e intermedios en la resina tipo poliéster, que evoluciona hasta un máximo de entalpía que produce un óxido multicomponente al sobrepasar los $210^{\circ} \mathrm{C}$ [26].

El tratamiento térmico continúa hasta que, en el caso de precursores tipo citrato, se inicia la formación de enlaces dobles $\mathrm{C}=\mathrm{C}$ con una transformación parcial del citrato a aconitato, y al mismo tiempo 
la formación de especies amoniacales comienza a evidenciarse entre 220 y $230{ }^{\circ} \mathrm{C}$. Luego, el sistema evoluciona a un evento exotérmico, relacionado con la eliminación de materia orgánica remanente del proceso de autocombustión entre 250 y $350{ }^{\circ} \mathrm{C}$, registrando pérdidas de un $5 \%$ en peso. En este punto, cuando la mayoría de la materia orgánica se ha destruido, inicia la eliminación de los carbonatos formados durante la etapa de síntesis, y comienza así la consolidación de la fase cristalina buscada. La naturaleza de los compuestos formados al final de esta etapa se han discutido en la literatura, y de acuerdo con esto, especies tipo carbonato, oxocarbonato y óxidos de especies intermedias se forman cuando se relacionan precursores tipo citrato, lo cual ha sido aceptado y confirmado por espectroscopia infrarroja y por datos de difracción de rayos $\mathrm{X}$, sugiriendo que estos intermedios siguen siendo amorfos incluso a $550{ }^{\circ} \mathrm{C}$ en una fase metaestable, que después de la eliminación de oxocarbonatos y demás precursores finaliza cuando la temperatura ha alcanzado los 580 ${ }^{\circ} \mathrm{C}$, como se indica en la figura 2 [27, 28]. En consecuencia, este análisis permitió evitar la densificación y la pérdida de área superficial por efectos de sinterización en los óxidos; cabe destacar que todos los precursores mostraron un comportamiento semejante con respecto a los valores de entalpía asociada a la descomposición de los precursores, por lo que no fue posible determinar desde esta técnica el efecto de la modificación del catión samario sobre el óxido de cerio.

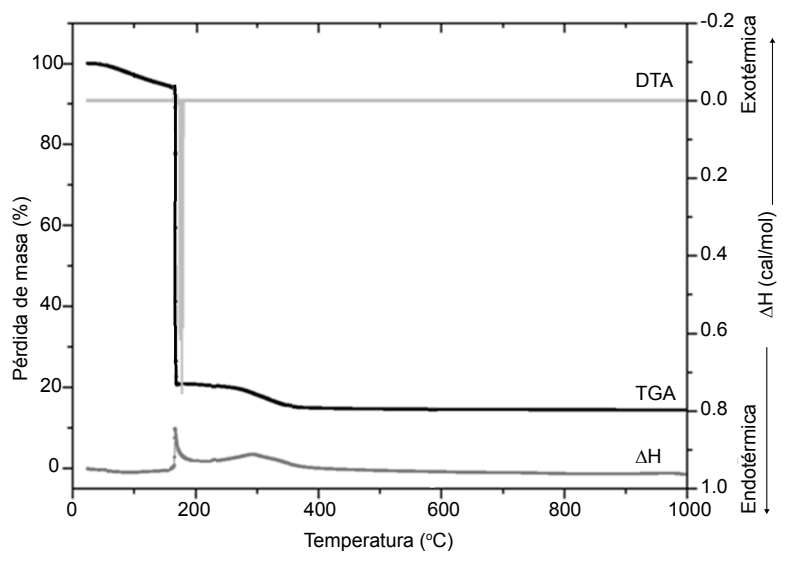

Figura 2. Análisis térmico gravimétrico y térmico diferencial del precursor SDC5.

La determinación de la composición en cada caso se realizó mediante microanálisis de energía dispersiva y fluorescencia de rayos $\mathrm{X}$, confirmando una buena correlación entre las composiciones propuestas y obtenidas, como se indica en la tabla 2; dichos resultados proporcionan información relacionada con la efectividad del proceso de síntesis en medio acuoso y favorecen el control efectivo en la composición. Las diferencias evidenciadas a nivel del catión samario en la serie de sólidos obtenidos se deben a que las muestras presentan un bajo nivel de modificación de este catión; sus valores se ajustan perfectamente a los límites de detección de las técnicas utilizadas en función de la homogeneidad microestructural de las muestras, resultando en una aparente reducción de la precisión.

Tabla 2. Comparación entre valores de composición propuesta y obtenidos para las muestras de $\mathrm{Ce}_{1-X} \mathrm{Sm}_{X} \mathrm{O}_{2}$ derivados del análisis por microsonda EDS y fluorescencia de rayos X.

\begin{tabular}{lcccc}
\hline Composición & $\mathrm{SDC} 0$ & $\mathrm{SDC} 5$ & $\mathrm{SDC} 10$ & $\mathrm{SDC15}$ \\
\hline Propuesta & $\mathrm{CeO}_{2}$ & $\mathrm{Ce}_{0,95} \mathrm{Sm}_{0,05} \mathrm{O}_{2}$ & $\mathrm{Ce}_{0,90} \mathrm{Sm}_{0,10} \mathrm{O}_{2}$ & $\mathrm{Ce}_{0,85} \mathrm{Sm}_{0,15} \mathrm{O}_{2}$ \\
Encontrada por EDS & $\mathrm{Ce}_{0,98} \mathrm{O}_{1,86}$ & $\mathrm{Ce}_{0,93} \mathrm{Sm}_{0,06} \mathrm{O}_{1,90}$ & $\mathrm{Ce}_{0,91} \mathrm{Sm}_{0,08} \mathrm{O}_{1,93}$ & $\mathrm{Ce}_{0,86} \mathrm{Sm}_{0,13} \mathrm{O}_{1,95}$ \\
Encontrada por XRF & $\mathrm{Ce}_{0,96} \mathrm{O}_{1,86}$ & $\mathrm{Ce}_{0,94} \mathrm{Sm}_{0,05} \mathrm{O}_{1,97}$ & $\mathrm{Ce}_{0,88} \mathrm{Sm}_{0,09} \mathrm{O}_{1,90}$ & $\mathrm{Ce}_{0,84} \mathrm{Sm}_{0,16} \mathrm{O}_{1,90}$ \\
\hline
\end{tabular}

$\mathrm{El}$ posterior análisis de difracción de rayos $\mathrm{X}$ indica que los sólidos de $\mathrm{Ce}_{1-X} \mathrm{Sm}_{X} \mathrm{O}_{2-\delta}$ tienen un tamaño de grano adecuado propio del método de síntesis, con una orientación cristalina preferencial en el plano cristalográfico (111), tal como se ilustra en la figura 3. La estimación del tamaño del cristalito se hizo utilizando las señales de difracción de mayor intensidad, usando la ecuación de Debye-Scherrer; el tamaño promedio de cristalito que se obtuvo fue de $41 \mathrm{~nm}$. La búsqueda realizada por el programa X'Pert High Score en las bases de datos de la ICCD condujo a una clasificación de fase acorde con un único grupo espacial y sistema cristalino. No existe evidencia de cambio en los parámetros estructurales de red del material por sustitución del catión lantánido, toda vez que el nivel de modificación se mantiene dentro de los límites de tolerancia establecidos para esta estructura.

El posterior refinamiento, indexación y simulación de la estructura de los óxidos de cerio se hizo a través del programa Cellref, bajo los parámetros antes mencionados, utilizando las 6 señales de mayor intensidad; esto permitió determinar que existen pequeñas diferencias en términos de las 


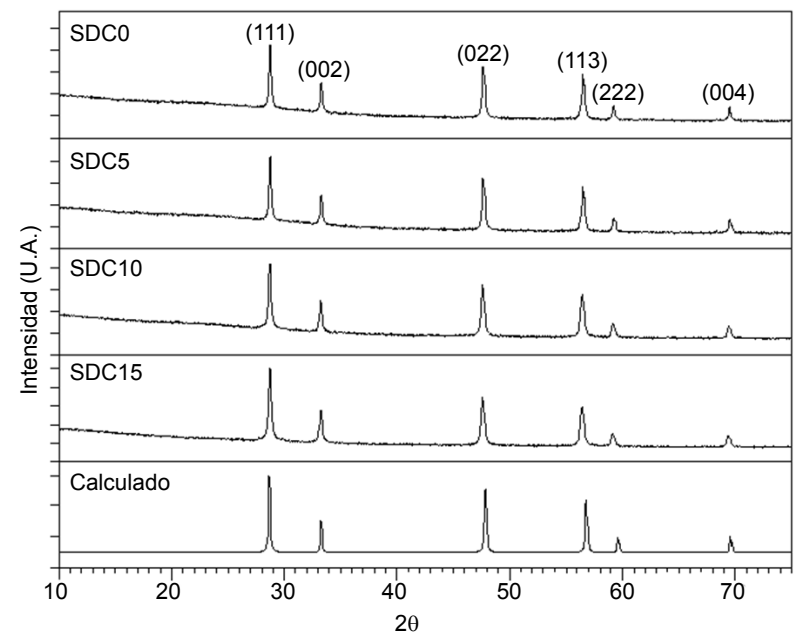

Figura 3. Patrón de difracción de rayos X, principales líneas de difracción indexadas y estructura calculada, para los sistemas SDC0, SDC5, SDC10 y SDC15.

características estructurales de cada sólido con respecto a la estructura cristalina de referencia del óxido de cerio, como se indica en la tabla 3. Dichos resultados están basados en una comparación isomórfica realizada por el programa de refinamiento de datos de difracción, y tan solo constituyen una herramienta analítica de comparación para verificar si existen cambios mayores en la serie de sólidos sintetizados. Ahora, si bien es cierto existe una diferencia en el tamaño del cristal y de parámetros de celda entre los diferentes sistemas, esta solo representa un estimado estadístico.

La morfología, la textura y las características superficiales de los sistemas $\mathrm{Ce}_{1-X} \mathrm{Sm}_{X} \mathrm{O}_{2-\delta}$ fueron evaluadas por técnicas microscópicas en muestras sin recubrimiento, como se muestra en la figura 4. Las micrografías confirman la obtención de un material con distribución de conglomerados heterogéneos; la morfología y la apariencia superficial de las partículas muestran que los sólidos están conformados por agregados irregulares multipartícula, distribuidos en forma heterogénea. En principio, esta presentación guarda relación con la textura y el relieve que se generan en los sólidos por la salida de sustancias volátiles que se producen en virtud de la degradación de los componentes orgánicos durante la autocombustión de las muestras y su posterior tratamiento térmico. Adicionalmente, es notable que los materiales hayan sufrido cierto grado de sinterización, hecho que favoreció la aparición de una morfología compacta que se reflejó en la intensidad de la reflexión (111). La textura y el relieve son característicos del método de síntesis utilizado, lo que evidencia que esta técnica confiere a los sólidos características relevantes en términos de superficie, área, tamaño y forma cristalina.

\begin{tabular}{lcccccc}
\multicolumn{7}{c}{ Tabla 3.Principales características estructurales de los sistemas $\mathrm{Ce}_{1-X} \mathrm{Sm}_{X} \mathrm{O}_{2-\delta}$. } \\
\hline Sistema & $\begin{array}{c}\text { Grupo } \\
\text { espacial }\end{array}$ & $\begin{array}{c}\text { Sistema } \\
\text { cristalino }\end{array}$ & $\begin{array}{c}\text { Volumen de } \\
\text { celda }\left(\AA^{3}\right)\end{array}$ & $\begin{array}{c}\text { Parámetros de } \\
\text { celda }(\AA)\end{array}$ & $\begin{array}{c}\text { Densidad } \\
\text { calculada }\left(\mathrm{g} . c m^{-3}\right)\end{array}$ & $\begin{array}{c}\text { Tamaño } \\
\text { cristalito (nm) }\end{array}$ \\
\hline $\mathrm{CeO}_{2}$ & $\begin{array}{c}F m-3 m \\
(225)\end{array}$ & Cúbico & 158,46 & 5,4113 & 7,215 & 39,0 \\
$\mathrm{Ce}_{0,95} \mathrm{Sm}_{0,05} \mathrm{O}_{2}$ & $\begin{array}{c}F m-3 m \\
(225)\end{array}$ & Cúbico & 157,79 & 5,4037 & 7,244 & 40,0 \\
$\mathrm{Ce}_{0,90} \mathrm{Sm}_{0,10} \mathrm{O}_{2}$ & $\begin{array}{c}F m-3 m \\
(225)\end{array}$ & Cúbico & 157,79 & 5,4037 & 7,244 & 40,0 \\
$\mathrm{Ce}_{0,85} \mathrm{Sm}_{0,15} \mathrm{O}_{2}$ & $\begin{array}{c}F m-3 m \\
(225)\end{array}$ & Cúbico & 158,45 & 5,4112 & 7,214 & 34,7 \\
\hline
\end{tabular}

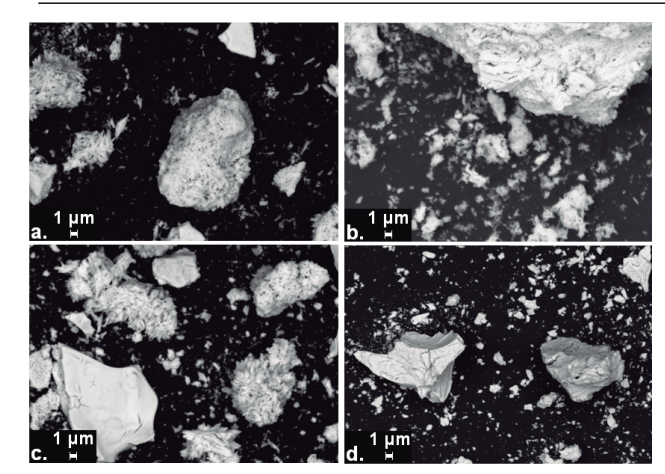

Figura 4. Micrografías electrónicas de barrido de las muestras a. SDC0; b. SDC5; c. SDC10 y d. SDC15.
El análisis de las muestras por microscopia electrónica de transmisión (figura 5) y el conteo estadístico de la estimación de los resultados tomando como referencia una población de 200 partículas, confirmaron la presencia de aglomerados de cristalitos nanométricos de forma regular en todas las muestras, con un tamaño promedio de $41 \mathrm{~nm}$ y distancias interplanares de $0.31 \mathrm{~nm}$ para el plano (111) y de 0.27 $\mathrm{nm}$ para el plano (002), acorde con investigaciones previas [29, 30]. 


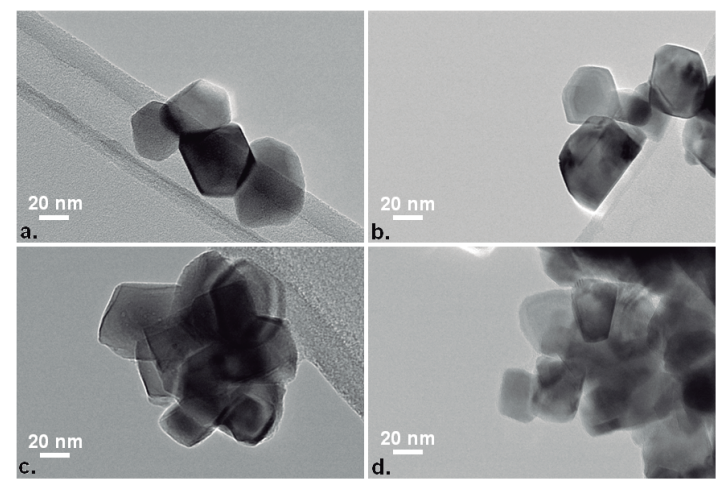

Figura 5. Micrografías electrónicas de transmisión de las muestras: a. SDC0; b. SDC5; c. SDC10 y d. SDC15.
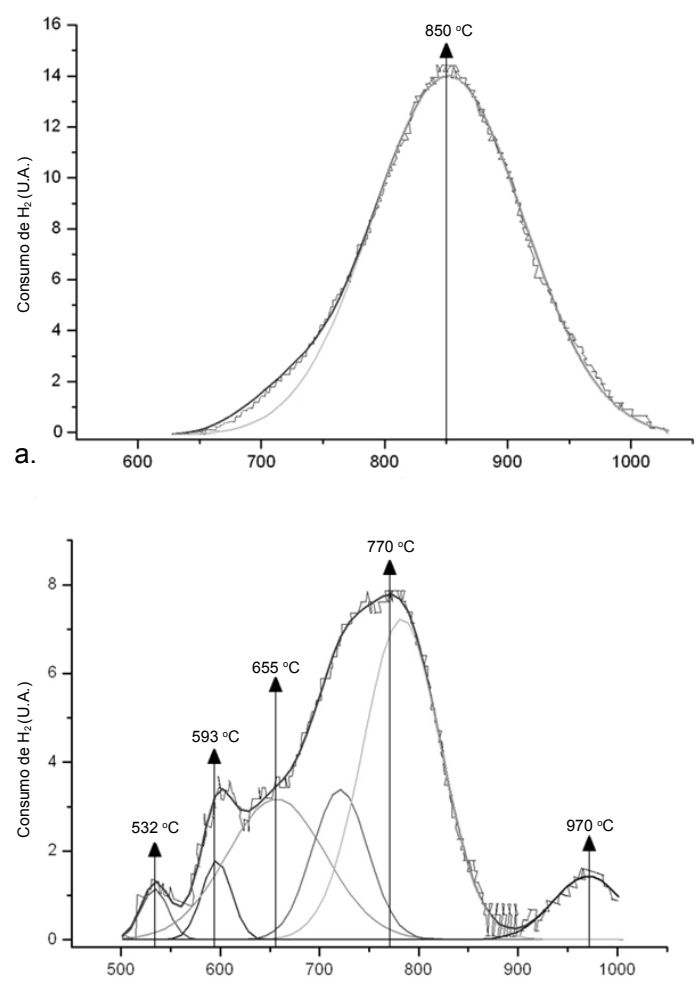

C.
El posterior análisis de reducción a temperatura programada permitió determinar que la cantidad de samario adicionado en las muestras afecta de forma proporcional la naturaleza del material, como se observa en la figura 6. Así mismo, la principal señal endotérmica de reducción se desplaza a regiones de menor temperatura, esto es: de $850{ }^{\circ} \mathrm{C}$ para la muestra sin adición de samario SDC0; $830^{\circ} \mathrm{C}$ para la SDC5; $770{ }^{\circ} \mathrm{C}$ para la SDC 10 , y $752{ }^{\circ} \mathrm{C}$ para la SDC15, indicando que la reacción se hace menos endotérmica, afectando las propiedades de los materiales en reacciones de oxidación bajo condiciones fuertemente reductoras [31].
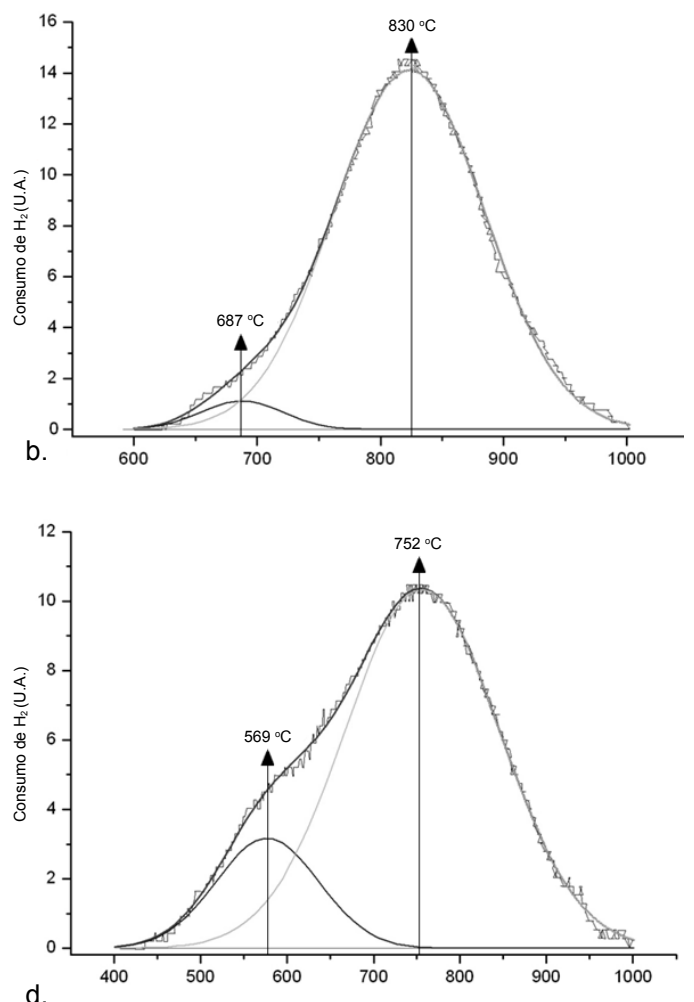

d.

Figura 6. Estudio de reducción a temperatura programada de las muestras a. SDC0, b. SDC5, c. SDC10 y d. SDC15.

Finalmente, en orden a determinar la microestructura eléctrica de los materiales, se obtuvieron datos de frecuencia variable, los cuales se muestran en forma de gráficas de impedancia tipo Nyquist; para esto, se obtuvieron cuatro pastillas de $0,0600 \mathrm{~g}$ de sólido en cada caso, que se prensaron isostáticamente al vacío $\left(10^{-5} \mathrm{mmHg}\right)$, a una presión de $14,0 \mathrm{MPa}$, por un periodo de 60 segundos; las pastillas así obtenidas se sinterizaron a $900{ }^{\circ} \mathrm{C}$ durante una hora, empleando una rampa de calentamiento de $5{ }^{\circ} \mathrm{C} \mathrm{min}^{-1}$, y se pulieron con ayuda de una lija de $\mathrm{SiC} 400$, con el fin de proporcionar una superficie homogénea para el proceso de electrodación, ajustando su espesor a $0,500 \mathrm{~mm}$. Las caras de la pastilla se recubrieron con ayuda de una aleación de indio y galio (1:1), suministrando una adecuada superficie de contacto. La medida y los datos de impedancia se corrigieron por la geometría global de las pastillas y de la celda de referencia. Los resultados se muestran en términos de la impedancia compleja entre el valor imaginario $\left(-\operatorname{Im} Z^{\prime \prime}\right)$ y el valor real $\left(Z^{\prime}\right)$, como se indica en la figura 7 , demostrando que los materiales reducen su conductividad eléctrica conforme la concentración del catión samario aumenta. Según algunas investigaciones [32, 33], el comportamiento de la conductividad eléctrica y la 
conductividad iónica son inversamente proporcionales, de tal manera que el incremento en la concentración de especies redox, como el samario, en la estructura básica del óxido de cerio permite favorecer el transporte de especies iónicas en el contexto de generar materiales de conductividad mixta iónica y electrónica (MIEC's) de creciente importancia en la industria de las pilas de combustible.

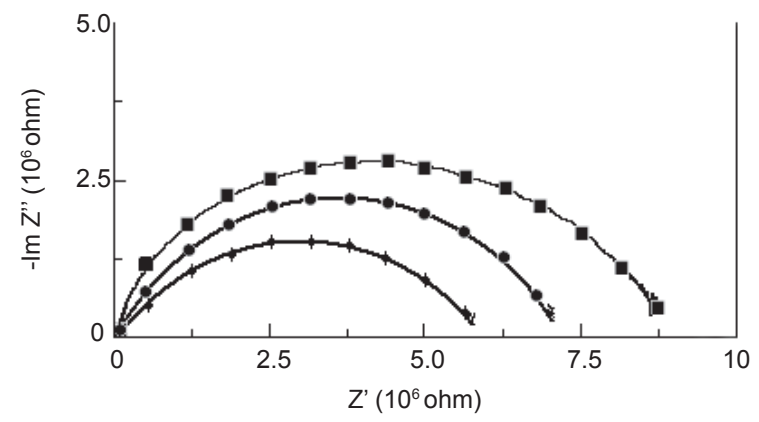

Figura 7. Graficas de impedancia tipo Nyquist que representan el comportamiento eléctrico de los sistemas SDC5 $\$$; SDC10 y SDC15 — medidos a temperatura ambiente.

Un análisis detallado, usando el programa Zview, demostró que la microestructura eléctrica del sólido es consistente con valores de resistividad globales de $8,9 \times 10^{6} \Omega$.cm para la muestra SDC15; de $6,9 \times 10^{6} \Omega$.cm para la muestra SDC10, y de $5,5 \times 10^{6} \Omega$.cm para la muestra SDC5; el correspondiente ajuste de la capacitancia en cada caso demostró que la relación existente entre la diferencia de potencial y el capacitor formado por el material y la carga eléctrica almacenada es considerablemente baja, del orden de $3,7 \times 10^{-12} \mathrm{~F}$ en todos los casos, indicando una tendencia a la reducción en el transporte de especies eléctricamente cargadas, lo cual favorece el transporte de especies iónicas [34]. Este efecto permite la movilidad de las principales especies formadas durante el proceso de oxidación del combustible $\left(\mathrm{H}_{2} \mathrm{O} \mathrm{CH}_{4}\right)$, reduciendo los fenómenos asociados a la pérdida de poder por activación, debido a la dificultad de transferir las especies químicas formadas cerca del área de contacto trifásica TPB (Triphasic Boundary). De esta forma, se pueden representar las muestras obtenidas por circuitos simples tipo RC en paralelo, consistentes con sistemas eléctricamente homogéneos que permiten separar la capacitancia y la permitividad de la frecuencia, con el fin de que esta última pueda mostrarse como una función lineal de la temperatura para permitir predecir el comportamiento de los materiales a las condiciones de operación de una celda de combustible de óxido sólido.

\section{Conclusiones}

En este trabajo queda demostrado que el método de polimerización-combustión con ácido cítrico permite la síntesis a baja temperatura de materiales nanométricos topotácticamente diseñados, no densificados y con estructura cristalina definida, basados en el sistema $\mathrm{Ce}_{1-X} \mathrm{Sm}_{X} \mathrm{O}_{2-\delta}$, con elevados niveles de pureza de fase, morfología y textura característica del método empleado, lo que permite obtener sólidos con características texturales, morfológicas y electrocatalíticamente activos para uso en pilas de combustible de óxido sólido. Los análisis de reducción a temperatura programada permitieron determinar que la cantidad de samario adicionado en las muestras afecta de forma proporcional la naturaleza del material, desplazando la señal endotérmica de reducción a regiones de menor temperatura y afectando la estabilidad del material final bajo condiciones reductoras.

Finalmente, el análisis microestructural utilizando espectroscopia de impedancias AC indicó que los materiales reducen su conductividad eléctrica conforme la concentración del catión samario aumenta, lo cual es un importante avance en la síntesis de materiales que presentan conductividad mixta iónicoelectrónica de creciente importancia en la industria de las actuales pilas de combustible, debido a que posibilitan el transporte de especies químicas cargadas iónicamente y formadas durante el proceso de oxidación del combustible $\left(\mathrm{H}_{2} \circ \mathrm{CH}_{4}\right)$. Por tanto, es claro que las herramientas analíticas aquí aportadas permitieron examinar de forma sistemática la promisoriedad de algunos materiales tipo fluorita bajo condiciones de sustitución por el catión, hecho que consolida un avance importante en la caracterización de nuevos componentes electrocatalíticos.

\section{Referencias}

[1] K. S. Weil, "The state of the art in sealing, technology for solid oxide fuel cells", JOM Overview, Fuel Cells, 2006. 
[2] H. L. Chum, R. P. Overend, "Biomass and renewable fuels", Fuel Process Technol, no. 71, pp. 187-190, 2001.

[3] S. H. Chan, O. L. Ding, "Simulation of a solid oxide fuel cell power system fed by methane", Int. J. Hydrogen Energy, no. 30, vol. 2, pp. 167179, 2005.

[4] S. Mukerjee, M. J. Grieve, K. Haltiner, M. Faville, J. Noetzel, K. Keegan, D. Schumann, D. Armstrong, D. England, J. Haller, C. De Minco, Electrochem. Soc. Proceedings. SOFC VII, no. 173, pp. 16-20, 2001.

[5] R. J. Gorte, J. M. Vohs, "Novel SOFC anodes for the direct electrochemical oxidation of hydrocarbons", J. Catal. no. 216, vol. 1-2, pp. 477-486, 2003.

[6] N. Minh, T. Takahashi, "Science and technology of ceramic fuel cells", Elsevier Science, Amsterdam, pp. 75, 1995.

[7] S. C. Singhal, "Recent progress in tubular solid oxide fuel cell technology", Electrochem. Soc. Proceedings, SOFC VII. no. 166, pp. 1-16, 2001.

[8] G. Hoogers, Fuel cell technology handbook, New York, Washington. pp. 10-120, 2003.

[9] W. Z. Nernst, "Über den mechanismus der elektrischen stromleitung im nernststift", Elektrochem, no. 6, pp. 41-50, 1899.

[10] M. Dokiya, "SOFC system and technology", Solid State Ionics, no. 152-153, pp. 383-392, 2002.

[11] R. Peters, R. Dahl, U. Kluttgen, C. Palm, D. Stolten, "Internal reforming of methane in solid oxide fuel cell systems", J. Power Sources, no. 106, pp. 238-244, 2002.

[12] Kyoto Protocol of the United Nations. Framework Convention on Climate Change. Report document. 1997.

[13] H. Kim, S. Park, J. M. Vohs, R. J. Gorte, "Direct oxidation of liquid fuels in a solid oxide fuel cell", J. Electrochem. Soc., no. 148, pp. A443-A446, 2001.
[14] C. Lu, W. L. Worrell, C. Wang, S. Park, H. Kim, J. M. Vohs, R. J. Gorte, "Development of solid oxide fuel cells for the direct oxidation of hydrocarbon fuels". J. Solid State Ionics, no. 393, pp. 152-153, 2002.

[15] C. Peng, Y. Zhang, Z. W. Cheng, X. Cheng, J. Meng, "Nitrate citrate combustión synthesis of $\mathrm{Ce}_{1-x} \mathrm{Sm}_{x} \mathrm{O}_{2-x / 2}$ solid solutions". J. Materials Science: Materials in Electronics, no. 13, pp. 757-762, 2002.

[16] V. Fernandes, R. Mossanek, P. Schio, J. Klein, A. de Oliveira, W. Ortiz, N. Mattoso, J. Varalda, W. Schreiner, M. Abbate, D. Mosca, "Dilute defect magnetism: origin of magnetism in nanocrystalline $\mathrm{CeO}_{2}$. J. Physical Review B., no. 80, vol. 035202, pp. 1-7, 2009.

[17] B. Bakiz, F. Guinneton, J. P. Dallas, S. Villain, J. R. Gavarri, "From cerium oxycarbonate to nanostructured Ceria: Relations Between Synthesis, Thermal Process and Morphologies". J. Crystal Growth, no. 310, pp 3055-3061, 2008.

[18] Puigdomenech, I. Make equilibrium diagrams using sophisticated algorithms Medusa. 2002. Royal Institute of Technology. Stocholm, Sweden.

[19] S. Tamilmani, J. Shan, W. Huang, S. Raghavan, R. Small, C. Shang, B. Scott, Interaction between Ceria and Hydroxylamine. EKC Technology, Inc., Hayward, CA. Materials Research Society Symposium. 2003.

[20] T. Enz, H. Sieger, C. Fasel, H. Hahnz, "Nanocomposite Formation Through Thermal Decomposition of Mixed Samarium and Magnesium Citrate-Derived Gels Formed by Spray Pyrolysis". J. Am. Ceram. Soc., no. 91, pp 30663073, 2008.

[21] H. Sieger, J. Suffner, H. Hahn, "Thermal Stability of Nanocrystalline $\mathrm{Sm} 2 \mathrm{O} 3$ and $\mathrm{Sm}_{2} \mathrm{O}_{3}$ MgO”. J. Am. Ceram. Soc., no. 89, pp 979-984, 2006.

[22] M. Popa, D. Crespo, J. M. Calderón-Moreno, "Synthesis and Structural Characterization of Single-Phase $\mathrm{BiFeO} 3$ Powders from a Polymeric Precursor". J. Am. Ceram. Soc., no. 90, pp. 2723-2727, 2007. 
[23] O. Carp, L. Patron, A. Ianculescu, D. Crisan, N. Dragan, R. Olar, "Influence of Precursor on the Thermally Induced Synthesis Dysprosium Manganite with Perovskite Structure". J. Thermal Analysis and Calorimetry, no. 72, pp. 253-261, 2003.

[24] Yu. Wang, J. Santiago-Avilés, "Synthesis of Lead Zirconate Titanate Nanofibres and the Fourier-Transform Infrared Characterization of their Metallo-organic Decomposition Process". J. Nanotechnology, no. 15, pp. 32-36, 2004.

[25] D. S. Todorovsky, M. M. Getsova, M. A. Vasileva, "Thermal Decomposition of Lanthanum Titanium Citric Complexes Prepared from Ethylene Glycol Medium". J. Mater. Sci., no. 37, pp. 4029-4039, 2002.

[26] S. G. Cho, P. F. Johnson, R. A. Condrate, “Thermal Decomposition of (Sr, Ti) Organic Precursors During the Pechini Process". J. Mater. Sci., no. 25, pp 4738-4744, 1990.

[27] D. Heninngs, W. Mary, J. Solid State Chem., no. 26, pp. 329-331, 1978.

[28] T. T. Carvalho, P. B. Tavares, "Synthesis and Thermodynamic Stability of Multiferroic $\mathrm{BiFeO}_{3}$ ". J. Materials Letters, no. 62, pp. 39843986, 2008.

[29] A. Hartridge, A. K. Bhattacharya, R. E. DuninBorkowski, J. L. Hutchison, "Homogeneity of Lanthanide Doped Ceria Nanocrystal Dispersions Using High-Resolution Transmission
Electron Microscopy". J. Nanoparticle Research, no. 3, pp. 433-441, 2001.

[30] S. Bernal, G. Blanco, J. J. Calvino, C. LópeCartes, J. A. Pérez-Omil, J. M. Gatica, O. Stephan, C. Colliex, "Electron microscopy (HREM, EELS) Study of the Reoxidation Conditions for Recovery of $\mathrm{NM} / \mathrm{CeO}_{2}$ (NM: Rh, Pt) Catalysts from Decoration or Alloying Phenomena". $J$. Catalysis Letters, vol. 76, no. 3-4, pp. 131-137, 2001.

[31] S. Zhao, R. J. Gorte, "The Effect of Oxide Dopants in Ceria on n-Butane Oxidation". J. Appl. Catal A: General, no. 248, pp. 9-18, 2003.

[32] M. Dudek, M. Mróz, L. Zych, E. Dro?d?Cie?la, "Synthesis of Ceria Based Nanopowders Suitable for Manufacturing Solid Oxide Electrolytes". J. Materials Science-Poland, vol. 26, no. 2, pp. 319-330, 2008.

[33] J. E. Shemilt, H. M. Williams, "Effects of Composition and Processing Method on the Low Temperature Conductivity of Samaria Doped Ceria Electrolytes". J. Materials Science Letters, no. 18, pp. 1735-1737, 1999.

[34] C. Mansilla, J. P. Holgado, J. P. Espinós, A. R. González-Elipe, F. Yubero, "Microstructure and Transport Properties of Ceria and Samaria Doped Ceria Thin Films Prepared by EBEIBAD". J. Surface $\mathcal{E}$ Coatings Technology, no. 202, pp. 1256-1261, 2007. 